\title{
Pregnancy in a patient with paroxysmal nocturnal hemoglobinuria
}

\author{
Anju Singh $^{1 *}$, Pooja Sikka ${ }^{1}$, Vanita Suri ${ }^{1}$, Neelam Agrawal ${ }^{1}$, Seema Chopra ${ }^{1}$, Bhupesh Kumar $^{2}$ \\ ${ }^{1}$ Department of Obstetrics \& Gynaecology, Post Graduate Institute of Medical Education and Research, Chandigarh, \\ Punjab, India \\ ${ }^{2}$ Department of Anesthesia and Intensive Care, Post Graduate Institute of Medical Education and Research, \\ Chandigarh, Punjab, India
}

Received: 01 February 2014

Accepted: 16 February 2014

\section{*Correspondence:}

Dr. Anju Singh,

E-mail: singha1712@gmail.com

(C) 2014 Singh A et al. This is an open-access article distributed under the terms of the Creative Commons Attribution Non-Commercial License, which permits unrestricted non-commercial use, distribution, and reproduction in any medium, provided the original work is properly cited.

\begin{abstract}
The occurrence of Paroxysmal Nocturnal Hemoglobinuria (PNH) during pregnancy is very rare. It can cause significant fetomaternal morbidity and mortality due to associated complement mediated hemolysis and/or thrombosis. We report a case of PNH in a pregnant lady who presented to our antenatal clinic at 10th weeks of gestation. Her pregnancy was managed with multiple blood transfusions and steroid administration. During 3rd weeks postpartum period she developed sepsis with acute renal failure and posterior reversible encephalopathy syndrome requiring prolonged hospitalization. She was subsequently discharged from hospital in satisfactory condition.
\end{abstract}

Keywords: Paroxysmal nocturnal hemoglobinuria, Pregnancy, Management

\section{INTRODUCTION}

Paroxysmal nocturnal hemoglobinuria $(\mathrm{PNH})$ is a rare hematological disorder. ${ }^{1}$ Its occurrence during pregnancy is very rare, but it can cause significant fetomaternal morbidity and mortality. ${ }^{2}$ The estimated maternal mortality range from $5.8 \%$ to $20.8 \%$, most common cause being thromboembolism. ${ }^{3}$ We report a case of PNH in a pregnant lady who presented to us at $10^{\text {th }}$ weeks of gestation for antenatal checkup. During pregnancy she had multiple haemolytic crises that were managed with multiple blood transfusions and steroid administration. At $3^{\text {rd }}$ weeks postpartum she developed sepsis, acute renal failure, and multiple related complications including rare posterior reversible encephalopathy syndrome (PRES).

\section{CASE REPORT}

The patient was 23 year old, third gravida with history of two previous first trimester abortion. She was a known case of PNH since 4 years when she had anemia with fever, breathlessness, easy fatigability and cola coloured urine. Her anemia was unresponsive of routine hematinics and she was referred to our institute's medical department where her anemia work up revealed acquired hemolytic anemia (hemoglobin $4 \mathrm{gm}$, total serum bilirubin- $4 \mathrm{mg} / \mathrm{dl}$, unconjugated fraction- $3 \mathrm{mg} / \mathrm{dl}$ together with positive sucrose lysis test, Ham's acid hemolysis (HAM test) and urine for hemosiderin. The Bone marrow examination showed hypercellularity with adequate iron stores while flow cytometry of CD55 and CD59 was positive in erythrocytes). She was managed with transfusion of blood together with steroid and danazol administration but she developed allergy to danazol requiring it to stop.

Before this index pregnancy, she had multiple episodes of hemolytic crisis requiring multiple blood transfusions. She was advised against pregnancy but conceived spontaneously and presented to our clinic for antenatal checkup at 10 weeks of gestation. Besides her routine antenatal care, she was prescribed with prednisolone 20 $\mathrm{mg}$ daily. However at 16 weeks of gestation her hemoglobin dropped to $4 \mathrm{gm}$ due to hemolytic crisis which required 3 units of packed red blood cells transfusion. The prednisolone dose was increased to 45 
$\mathrm{mg}$ daily and aspirin was started at dose of $75 \mathrm{mg}$ once daily to prevent thrombosis. She did not receive any anticoagulant during antenatal period. Her platelet counts remained within normal range throughout the pregnancy but she had hemolytic crisis requiring transfusion of several units of blood at 32 and 34 weeks of gestation. She had asymmetrical intrauterine growth restriction (IUGR) at 32 weeks of gestation. At $35+4$ weeks of gestation she was admitted to the hospital for safe confinement for moderate anemia (Hb-8gm) and IUGR (EFW-1.8 kg). Her routine work up suggested mild preeclampsia (BP-140/90 $\mathrm{mmHg}, 24 \mathrm{hr}$ urinary protein$560 \mathrm{mg}$ ) and was managed conservatively.

At 36 weeks of gestation she had preterm rupture of membrane. Induction of labour was done with oxytocin and labour analgesia was achieved with tramadol. She delivered a live $1.7 \mathrm{~kg}$ girl vaginally with APGAR score of 8 and 9 at 1 minute and 5 minute respectively. Immediately after delivery she was prescribed low molecular weight heparin at rate of $1 \mathrm{mg} / \mathrm{kg}$ subcutaneously as prophylactic measure for thrombosis. She was discharged from hospital on $5^{\text {th }}$ postpartum day in a satisfactory condition.

At $3^{\text {rd }}$ postpartum weeks she returned back to our hospital with history of fever with chills, acute abdominal pain and decreased urine output. Blood investigation showed features of sepsis (total leucocyte count-24,000, platelets37,000 , hemoglobin-6.2 gm) associated with acute renal failure (blood urea-139, serum creatinine- 4.1), chest $\mathrm{x}$ ray showed abscess in the left lung, while computed tomography abdomen showed liver and spleen infarct. She was managed conservatively with intravenous antibiotics, fluid and diuretics. Two days after admission to the hospital she had raised blood pressure (180/110 to $200 / 130 \mathrm{~mm}$ of $\mathrm{Hg}$ ) followed by seizure. This was managed with infusion of nitroglycerine, magnesium sulphate and levetiracetam. Magnetic resonance imaging (MRI) of brain revealed hyperintensities in bilateral cerebral and cerebellum suggestive of PRES and right lobe infarct. Patient was discharged on postpartum day 40 in satisfactory condition on prednisolone and antibiotics. At follow up visit she is doing well on steroids.

\section{DISCUSSION}

$\mathrm{PNH}$ is an acquired hemolytic anemia due to defect of glycosylphosphatidylinositol (GPI) protein in the cell membrane of bone marrow stem cells. It causes increased sensitivity of red cells to complement leading to intravascular hemolysis and hemoglobinuria. Other associated feature includes cytopenia and increased frequency of thrombotic events. Since most patients of PNH have both normal and abnormal protein deficient RBC, it usually does not manifest fully until young adulthood, lessen the symptom and causes delay in diagnosis. Recently developed flow cytometry assays of GPI-anchored protein allow screening for PNH. Flow cytometry is more sensitive than traditional Ham's test and sucrose lysis test. In our case Ham's test, sucrose lysis test and flow cytometry of CD55 and CD59 on red and white blood cells were positive confirming the diagnosis of PNH.

The maternal complications are seen in around three quarters of cases with $10 \%$ maternal mortality and fetal loss in about $40 \%$. Hemolysis and thrombosis are the most frequent cause of fetomaternal morbidity and mortality. ${ }^{4}$ The episodes of hemolytic crisis are triggered by stresses. ${ }^{5}$ Resulting anemia may be severe during pregnancy due to superadded $\mathrm{PNH}$ induced hemolytic crisis on preexisting pregnancy induced physiological anemia. It often warrants blood transfusion as seen in our patient.

Thrombosis, occurs commonly during post-partum period $(30.2 \%)$ than antenatal or intrapartum period $(16.2 \%){ }^{6}$ Hepatic vein thrombosis (Budd-Chiari syndrome) is the most common thrombotic complication associated with pregnancy with a peak incidence in the post-partum period. $^{7}$ To prevent thrombosis event we used LMW heparin immediately after delivery. Inspite of this our patient had liver, splenic and frontal lobe infarct. However whether these were thrombotic or infective in origin we are not sure.

The infections rate is disproportionately high in cases of PNH. ${ }^{8}$ Lack of GPI-anchored effectors molecules in granulocytes and monocyte in PNH may be predisposing factor for this. This predisposition together with use of steroid therapy may have been the cause for sepsis and lung abscess in our case.

Acute renal failure seen in $\mathrm{PNH}$ is due to hemosiderin deposition in kidney tubular cells. Under normal circumstances, hemosiderin deposited is mildly toxic but in the presence of low circulating blood volume and aciduria it can induce acute renal failure. ${ }^{9}$ Our patient presented with high grade fever together with less oral fluid intake leading to reduction in circulating fluid volume thus predisposing hemosiderin induced acute renal failure. We managed her renal dysfunction by increasing circulating fluid volume, use of diuretics and together with steroid to reduce complement mediated hemolysis.

PRES is a rare neurologic disorder characterized by acute increase in blood pressure and by headache, seizure and altered mental status. Risk factors for PRES include malignant hypertension, eclampsia, medications such as immunosuppressant, renal failure and chemotherapy. ${ }^{10}$ Our subject had seizure which was part of PRES as seen on MRI which was possibly predisposed by renal failure and accelerated hypertension.

Treatment for PNH includes blood transfusions, not only to increase $\mathrm{Hb}$ concentration but also to suppress marrow production of complement sensitive erythrocyte during an episode of sustained hemoglobinuria. Our patient had severe anemia ( $\mathrm{Hb}-4$ to $7 \mathrm{gm}$ ) throughout her pregnancy 
even though we targeted $\mathrm{Hb}$ level of $10 \mathrm{gm}$ using multiple transfusion. Low hemoglobin can be associated with low birth weight. In spite of multiple blood transfusion our patient had an IUGR and preterm baby. In a review by Ray et al. almost half of all infants delivered were preterm and growth retarded. ${ }^{4}$ Other treatment modalities in PNH include oral prednisolone and androgen therapy (danazol) for persistent or severe hemolysis. Danazol is contraindicated during pregnancy due to its teratogenic effects.

Pregnancy in a patient with PNH can be life threatening and is not recommended. Even if it occurs, it should be followed closely by both obstetricians and hematologist in a tertiary care setting to avoid multiple complications.

Funding: No funding sources

Conflict of interest: None declared

Ethical approval: Not required

\section{REFERENCES}

1. Higgins SP, McMahon LP, Brennecke SP. Paroxysmal nocturnal haemoglobinuria in pregnancy-not to be confused with preeclampsia or HELLP syndrome. Case report and literature review. J Obstet Gynaecol. 2004;24:83-5.

2. Packman $\mathrm{CH}$. Pathogenesis and management of paroxysmal nocturnal haemoglobinuria. Blood Rev. 1998;12:1-11.

3. Bais J, Pel M, Von dem Borne A, van der Lelie H. Pregnancy and paroxysmal nocturnal hemoglobinuria. Eur J Obstet Gynecol Reprod Biol. 1994;53:211-4.
4. Ray JG, Burows RF, Ginsberg JS, Burrows EA. Paroxysmal nocturnal hemoglobinuria and the risk of venous thrombosis: review and recommendations for management of the pregnant and non-pregnant patient. Haemostasis. 2000;30:103-17.

5. Chanana C, Sharma JB, Malhotra N, Kumar S, Kumar K. Successful maternal and fetal outcome of a patient with paroxysmal nocturnal hemoglobinuria in pregnancy: a case report. Calicut Medical Journal. 2007;5:e4.

6. Fieni S, Bonfanti L, Gramellini D, Benassi L, Delsignore R. Clinical management of paroxysmal nocturnal hemoglobinuria in pregnancy. A case report and updated review. Obstet Gynecol Surv. 2006;61:593-601.

7. Chen ML, Yu CH, Chang FM, Kuo PL. Paroxysmal nocturnal hemoglobinuria superimposed with preeclampsia. Taiwanese J Obstet Gynecol. 2006;45:276-9.

8. Bjorge L, Ernst P, Haram KO. Paroxysmal nocturnal hemoglobinuria in pregnancy. Acta Obstet Gynecol Scand. 2003;82:1067-71.

9. Qi K, Zhang XG, Liu SW, Yin Z, Chen XM, Wu D. Reversible Acute Kidney Injury Caused by Paroxysmal Nocturnal Hemoglobinuria. Am J Med Sci. 2011;341:68-70.

10. Nuwer JM, Eshaghian S. Late post-partum eclampsia with posterior reversible encephalopathy syndrome. Hospital Physician. 2007;43(6):45-9.

DOI: $10.5455 / 2320-1770 . i j r \operatorname{cog} 20140365$

Cite this article as: Singh A, Sikka P, Suri V, Agrawal N, Chopra S, Kumar B. Pregnancy in a patient with paroxysmal nocturnal hemoglobinuria. Int J Reprod Contracept Obstet Gynecol 2014;3:285-7. 